\title{
Les territoires imaginaires de l'altérité: divers aspects de la frontier dans les Amériques
}

\section{Alterity and Imaginary Territories: Multiple Aspects of the Frontier in the Americas}

Patrick Imbert ${ }^{1}$

Submetido em 24 de dezembro de 2016 e aprovado em 19 de janeiro de 2017.

Résumé: On étudiera la géosymbolique de la frontière et de la frontier et leur relation aux altérités minoritaires. Dans deux livres similaires publiés par le musée des civilisations on parle, en anglais, des «Inuit communities were real communities not merely groups of people who happened to be living near one another». Dans le livre en français, les Inuits «évoluent à l'intérieur d'une terre d'errance... au-delà d'une certaine limite, danger». Chacun invente son minoritaire en fonction de ses angoisses identitaires liées à une défense des frontières. De nos jours toutefois, les territorialités symboliques même dans l'espace exigu du radeau dans L'histoire de Pi transforment la frontière en frontier par la domestication des peurs entre cultures autres. Ce réseautage domine ainsi l'immensité spatiale menaçante.

Mots-clefs: Frontier/frontière. Métissage. Réseautage. Amériques.

\begin{abstract}
In order to analyze the situation of minorities, we will study the geo-symbolic aspects of the frontier, which is representing a non-finite space. It will be opposed to the border and its enclosed perspective. In two similar books published by the Museum of Civilization, one in English and one in French, Inuit Communities are presented in very different ways: In English, «Inuit Communities were real communities not merely groups of people who happened to be living near one another». In the French book, Inuits «move within a non-defined space... beyond a certain limit: danger!» Anglo-Canadians and French-Canadians invent their own minorities depending on their specific identities and their relationship to limits. Today, however, symbolic territorialities, even within the confine of a raft such as in Life of $P i$, transform borders into the frontier thanks to the taming of deep anguishes linked to encounter with alterity. Encounters are creating a new network of sharing and help control the immensity of any geo-symbolic territory.
\end{abstract}

Keywords: Frontier/border. Hybridity. Networking. The Americas.

\section{Introduction}

Les espaces des Amériques ont été perçus comme des espaces immenses et vides. C'est pour cela qu'ils ont attiré des millions d'immigrants qui ont pu s'inventer une vie 
nouvelle. De plus, dans certains pays, ces espaces ont été cadastrés et donnés ou vendus pour très peu afin qu'ils soient mis en valeur. Dans ces pays, les gens qui s’y sont installés ont obtenu des titres de propriété base d'une économie qui progressera vite et qui pourra créer une classe moyenne, car ces titres représentent des garanties pour obtenir du crédit et investir dans des entreprises plus ambitieuses. C'est d'ailleurs ce qu'affirme Hernando de Soto dans The Other Path (SOTO, 1989). Il critique la bureaucratie péruvienne qui étouffe tout esprit d'entreprise et qui condamne la plupart des gens à vivre dans le commerce informel.

Autrement dit, l'espace n'est pas une question de nature mais bien de culture et en particulier de culture économique et politico-légale. Elle implique dans tous les groupes et à toutes les époques des stratégies de coopération afin de tirer le meilleur parti de ressources qui sont finies comme toute ressource liée à une territorialité. Pourtant, comme nous le verrons, la force de l'espace des Amériques et sa particularité est qu'il a été imaginé comme immense et même comme non-fini. C'est le mythe de l'Ouest ou du Grand Nord et celui de la Pampa ou du Sertão. Dans ce cas, la territorialité devient un moyen de dominer pour un temps la contradiction entre monde fini et monde non-fini.

\section{La frontier et la Garrison mentality}

Son rêve était plus excitant que sa mémoire, voilà le grand principe sur lequel s'est édifiée l'Amérique.' dit l'assistant de recherche de Martin quand il cherche à savoir ce qu'est devenu Dubois, un Canadien français parti dans l'Ouest (CARRIER, 1996, p. 207).

La frontier tente d'échapper au dualisme propre à la logique territoriale. Elle en est sa sortie paradoxale abondamment racontée dans tous les récits du Wild West propres aux États-Unis. En effet, la frontier est un espace géographique présenté comme vide, ouvert et surtout sans fin, ce qui n'est jamais le cas d'un espace géographique par opposition au savoir qui, lui, peut être pensé comme non-fini² ${ }^{2}$ Cette narrativité paradoxale liée à la frontier est ce qui fonde la rupture d'avec un imaginaire européen qui repose, en particulier depuis le traité de Westphalie menant à la volonté de créer des États-Nations stables et délimités, sur des frontières, donc sur des espaces restreints, enfermés sur eux-mêmes et dont l'expansion ne peut se concevoir que par la guerre. En effet, face à cette somme 
finie de richesses qu'est tout territoire, pour prendre de l'expansion économique et il faut attaquer l'autre. Dans ce cas, si l'un gagne l'autre perd en une constante réactivation des effets de la croyance que la vie est un jeu à somme nulle (IMBERT, 2013).

Au Canada, longtemps mêlant des éléments de cultures européennes et de cultures des Amériques, on retient l'ambiguïté du concept de frontier, notamment chez W. L. Morton qui est fasciné par le Nord. Dans son texte intitulé «The North in Canadian Historiography», à un endroit, le terme frontier signifie plutôt une limite: "As the agricultural frontier moved westward, it left an open flank to the north. This became a permanent frontier, an enduring demarcation line between wilderness and farmland, between North and South» (MORTON, 1980, p. 234)3. Toutefois, à la fin de son texte, c'est un espace non fini: «Finally, the North... as a source of treasure, ... the unfolding of the permanent frontier $\left.{ }^{4}\right\rangle$ (Ibid., p. 239). C'est là, selon lui, que réside l'avenir du Canada, de ses richesses et du pouvoir du Gouvernement fédéral. On voit, dans son texte, le syncrétisme culturel à l'œuvre entre la conception européenne du territoire, le Nord comme limite à l'agriculture céréalière et, en même temps, le Nord comme lieu ouvert au développement quasi non fini de richesses grâce aux ressources et à la technologie.

Cette frontier est une catégorie importante pour comparer les Amériques entre elles, car elle peut être métaphysique comme on le voit dans la littérature brésilienne, lieu d'ordre économique comme au Canada ou d'anarchie économique comme aux ÉtatsUnis. Ainsi, concevoir l'arrière-pays comme immense est une chose, mais le concevoir comme barbare ajoute une dimension d'exclusion. Très tôt, en effet, on a reconnu que le territoire n'était pas vide. Il était peuplé. Le progressisme positiviste et civilisateur fondé sur la domination de l'espace naturel par le savoir et la technologie a donc dû inventer ceux qui étaient déjà là selon des paradigmes qui légitimaient leur exclusion. Ils étaient des barbares non-civilisables comme l'a souligné Sarmiento dans son essai intitulé Facundo $(1845,1934)$. Ils ont même été capturés et emmenés au jardin d'acclimatation à Paris en 1889 ou dans d'autres grandes villes européennes pour être présentés dans des zoos humains (BAEZ; MASON, 2006). Cette perspective d'origine européenne invente une frontière intérieure qui coupe le pays en deux, qui pose un espace menaçant, qui fait qu'on tend à se réfugier dans la ville tournée vers l'Europe perçue comme civilisée tandis 
que l'arrière-pays est barbare et parfois d'une barbarie d'où proviennent les dictateurs comme Rosas l'Argentin rejeté par Sarmiento. Toutefois, cette ville tournée vers l'Europe et ses canons que l'on doit imiter tend à avoir un impact colonial retardant les innovations provenant du nouveau continent. C'est ce qu'a souligné, déjà en 1928, le brésilien Oswald de Andrade dans son Manifeste anthropophage (1928; 1982). C'est ce que problématise, d'une autre manière en insistant sur le besoin de protection face à l'immensité, Margaret Atwood qui critique la garrison mentality. Elle se fonde sur le fait que, dans la littérature canadienne anglaise jusqu'aux années 1960, l'individu, surtout s'il est immigrant, est montré comme échouant souvent au Canada tandis que collectivement, comme on le voit chez Pierre Berton dans The National Dream ou The Last Spike, il tend à réussir. Comme le dit Margaret Atwood: «The collective hero can be an expression of a closed and ingrown garrison mentality or of a living community; collective action has been necessary for survival but it may also stifle individual growth» $(1972 \text {, p. 172) })^{5}$. Cette remarque rejoint W.L. Morton, l'avocat d'une histoire du Nord marquant le Canada: «The North makes necessary an absolute dependance on one's fellows on co-operative skills, on communal capital» (MORTON, 1980, p. 239) . Ainsi, la garrison mentality est une expression de nécessité de protection face à un espace barbare qui pose un frein à l'expansion. Cette protection, face à la vaste nature ou face aux altérités menaçantes comme les Autochtones, se recontextualise jusqu'à nos jours par le truchement de conversations stéréotypées sur le froid qui menace et qui crée un point de départ pour un dialogue stéréotypé ou même une coopération possible. Cela est souligné aussi par la narratrice de Tres lotos en un mar de fuego de Camila Reimers: «Conversación ideal en ascensores, fiestas de diplomáticos y comienzo de reuniones de negocios. Mientras más frío mejor, nos sentimos unidos por una naturaleza inhóspita, héroes compartiendo un destino congelado» ${ }^{7}(2006$, p. 97).

\section{La frontier: les populations d'origine européenne et les Autochtones}

La frontier différencie les cultures dans l'invention de soi et dans l'approche aux autres. Certes, elle se conçoit comme espace vide et ouvert. Elle efface l'expulsion et le massacre des Autochtones. Il faut retenir toutefois que la conception d'un espace ouvert est liée aux cultures autochtones comme le souligne Jean-Paul Lacasse. Il étudie 
les cultures innus du Canada et note que le rapport au territoire est différent de celui des Européens, car, pour le chasseur innu, le « territoire était partout» (2004, p. 153). Cette conception du territoire comme frontier, comme espace ouvert, est l'opposé des angoisses européennes qui, comme le soulignent les Innus, placent des frontières partout et ont divisé le territoire pour leurs fins.

De ce point de vue, il est intéressant de noter aussi comment un autre groupe, les Inuits du cuivre, sont représentés dans deux versions du même livre, l'une en anglais et l'autre en français, par le Musée des civilisations, maintenant le Musée de l'histoire à Ottawa. On y voit des différences importantes dans la manière dont les anglophones et les francophones construisent les Inuits du cuivre et surtout la perspective québécoise minoritaire face à la menace de l'espace lointain appartenant aux autres. Dans le livre en anglais, le groupe est organisé autour de l'indépendance des familles et des individus. Ils sont liés à des groupes régionaux ce qui fait qu'ils explorent des territoires immenses : «Inuit communities were real communities not merely groups of people who happened to be living near one another» (MORRISON; GERMAIN, 1995, p. 32). Dans le livre en français, les Inuits «évoluent à l'intérieur d'une terre d'errance que la tradition et d'ancestrales querelles ou de vieilles peurs ont vaguement délimitée [...] au-delà d'une certaine limite [...] Danger» (GERMAIN, 1995, p. 32). Ainsi, le présentateur québécois projette sa conception du territoire et de la menace que représente l'autre dans un contexte minoritaire face à la mer anglophone. Comme les Inuits du cuivre, la culture innue, (à ne pas confondre avec celle des Inuits), voit plutôt, selon Jean-Paul Lacasse, le territoire comme espace immense, comme frontier qui n'est pas une menace pour le soi intérieur. Pour les Québécois, par contre, l'au-delà de la limite provinciale représente le monde anglophone qui est assimilateur et rend les Francophones minoritaires. L'au-delà de la limite est donc un espace culturellement et même économiquement menaçant. Le commentateur francophone a donc tendance à interpréter le fonctionnement des Inuits du cuivre comme similaire à ses propres aprioris, donc à penser à la fois en fonction d'une durée longue couplée à un espace restreint. Cette perspective rappelle plus l'Europe que les Amériques qui tendent, elles, à penser en termes de durée courte qu'il faut compenser 
(BOUCHARD; LACOMBE, 1999) couplée à un espace immense à réseauter comme l'a rêvé Marshall McLuhan, échappant par la technologie à la garrison mentality.

\section{Frontier et réseautage}

La frontier pensée dans toute la force d'expansion de sa logique paradoxale, permet ainsi de repousser indéfiniment les Autochtones, mais surtout d'offrir un espace de richesses présentées comme un potentiel infini aux millions d'immigrants qui rêvent de devenir des settlers, des self-making men et d'être propriétaires. La frontier est le lieu de jonction aux États-Unis ainsi que dans l'Ouest canadien ou dans le Grand Nord d'une liberté individuelle plus grande qu'en Europe ou que dans l'Est du continent, car elle permet de se développer en prenant de l'expansion. On y ressent une démocratie pratique couplée au capitalisme qui mesure et cadastre l'immensité et accorde des titres de propriété, ce qui ultimement aboutit à ce que la frontier, comme espace ouvert, devienne un espace plein et délimité comme tous les autres. Le pouvoir de la contradiction créatrice, celle de l'espace non fini de la frontier, est fort dans les Amériques qui ne cherchent pas à résoudre les contradictions, mais à accumuler les éléments comme on le voit lorsque, par exemple, François, le personnage de La Nuit de Ferron s'affirme comme communiste, bouddhiste et gérant de banque. On pense aussi à Piscine dans L'histoire de Pi de Yann Martel qui affirme qu'il est à la fois Chrétien, Musulman et Bouddhiste. Mais ce pouvoir créateur de la frontier n'a qu'un temps. Elle se résorbe dans la logique dominante de la propriété qui est d'ailleurs ce que cherche l'immense majorité des immigrants, même si est visé discursivement l'établissement d'une civilisation nouvelle et d'un développement économique en mouvement comme l'évoquait Frederick Jackson Turner: «The West opened a refuge from the rule of established classes, from the subordination of youth to age, from the sway of established and revered institutions $\rangle^{8}$ (TURNER, 1994, p. 37). Cet espace accueille d'ailleurs des individus souvent différents ou rejetés, non seulement les Mormons qui, persécutés, s'établissent dans les déserts de l'Utah, mais aussi quelques Noirs qui, d'esclaves dans le Sud, deviennent propriétaires. C'est ce que l'on constate dans l'ouvrage d'Oscar Micheaux intitulé The Conquest: The Story of a Negro Pioneer (1913). Voilà qui a un impact sur la construction identitaire, car les individus ne font pas 
qu'appartenir à un lieu, ils s'appartiennent, ce qui est révolutionnaire au $19^{\mathrm{e}}$ siècle, en possédant une propriété qui permet ensuite, car elle représente une garantie, d'obtenir du crédit et de développer le commerce. D'ailleurs en Argentine, Sarmiento devenu Président de la République, après son voyage en Europe, puis après son voyage au Canada et aux États-Unis qu'il trouve bien supérieurs à l'Europe, fait tout pour faire venir des immigrants, mais des immigrants plus «civilisés» que d'autres. Ce sont des settlers provenant du nord de l'Europe, de l'Allemagne, de la Suisse, de l'Angleterre, à qui on accorde des terres enregistrées dans des cadastres (RIZZO, 2003, p. 143-166) ${ }^{9}$. La pampa ouverte, cette frontier du cône sud se civilise comme la province d'Entre Rios par exemple. Elle se transforme en lieu de développement et d'intégration où des gens de partout coïncident sur un territoire nouveau ${ }^{10}$ où ils inventent de nouvelles relations socioéconomiques, mais toujours en excluant ou en génocidant Noirs et Autochtones.

\section{La frontier et la Destinée manifeste}

Si le Sertão brésilien est avant tout un espace métaphysique auquel n'est pas lié un développement économique appuyé sur une démocratie et une légalité puisque le Brésil, dirigé par un empereur, ne supprime qu'en 1883 l'esclavage, la frontier aux États-Unis est un espace économique et démocratique qui fait partie d'un récit plus large, celui de la Destinée manifeste (IMBERT, 2007, p. 179). Elle est pensée par John O’Sullivan en 1845, mais se pense déjà en 1818 quand le Président Andrew Jackson réussit à vaincre les forces conjuguées des Espagnols qui colonisent la Floride, et des Séminoles. Son but est de libérer ce territoire de l'horrible colonisation espagnole pour l'ouvrir à la démocratie et donc de réaliser le plan (au sens de design) de Dieu par le biais des ÉtatsUniens récompensés par Dieu pour leur travail et leur désir d'universaliser la démocratie afin d'éliminer la barbarie. Ce plan est lisible à travers une nature qui est, comme en Argentine ou au Canada au $19^{\mathrm{e}}$ siècle, inventée à partir d'un territoire littéraire dans des descriptions empruntées à des modes de pensée européens. La nature est alors une espèce de livre (AREA, 2006) qui guide la pensée économique des dirigeants. Mais il y a des différences entre les pays, car, au lieu de se concentrer sur les déserts peuplés de barbares comme Sarmiento en Argentine ou de Gaspé au Canada français, Alsop parle du 
Maryland comme d'un nouveau paradis terrestre où les fruits et les fleurs étaient comme les «hiéroglyphes de notre situation primitive, adamique» (DELUMEAU, 1992, p. 153). Cette vision s'accorde à la perspective des protestants et des déistes francs-maçons étatsuniens qui voient dans le plan de l'univers, la source d'une religion naturelle, celle dont parle Willliam Paley en 1804 dans Natural Theology (1809). Elle est gouvernée par un grand architecte qui a placé les Amériques, et les États-Unis en particulier, au cœur d'une mission civilisatrice. Comme l'affirme le Président Jackson: «Providence has showered on this favoured land blessings without number and has chosen you as the guardians of freedom to preserve it for the benefit of the human race» ${ }^{11}$ (FRESONKE, 1999, p. 6). On voit là aussi que le you est tourné vers l'avenir, comme la frontier, car elle concerne ce que l'on pourra développer. Le you rejoint les futurs immigrants plus l'humanité entière aspirant à la liberté. La frontier devient ainsi un jardin, un village puis un espace où s'implantent les valeurs de la modernité urbaine jusqu'au bungalow de banlieue, le savoir, l'industrie, la technologie ce qui se produit aux États-Unis, au Canada, en Argentine, etc. Pour le Président Jackson, la frontier géographique se combine à une frontier humaine, l'infini de l'humanité à venir maîtrisant de plus en plus l'expansion des savoirs non seulement économiques et technologiques, mais aussi culturels et éthico-démocratiques.

\section{Barbarie/civilisation, frontier}

La frontier brutale et sauvage comme on la voit souvent en Europe ou même au Canada par le biais du cow-boy qui se défend sans l'aide de la police ou de l'armée, est en fait civilisée économiquement et culturellement. Elle est le lieu de rencontre, certes brutal mais aussi ouvert à la coopération, de communautés diverses où chaque individu a décidé de prendre sa vie en main comme on le voit dans Hija de la fortuna d'Isabel Allende (1999) au sujet de la ruée vers l'or en Californie ou encore au Klondyke chez Jacques Languirand (1971). Dans ce texte, l'auteur élabore une réflexion duelle au sujet des deux tendances fortes qui dominent au Québec, celle du sédentarisme et celle du nomadisme. Les germes d'une classe moyenne ambitieuse et dynamique se mettent en place dans ces configurations qui déplacent le paradigme barbarie/civilisation vers un troisième terme, la frontier dont le développement le plus évident est la capitalisation des savoirs, car ceux- 
ci, contrairement au territoire, sont non finis. Cette dynamique se veut incluante, comme le soulignent Isabel Allende et Jacques Languirand, car elle tend à échapper à une vision de la vie comme jeu à somme nulle liée à une somme finie de richesses exacerbant les rivalités. On le saisit pour la vie des fermiers de l'Ouest durant la crise de 1929 comme le montre Sharon Butala: «This is how it was that my sisters and I grew up with the notion of the farm as a mythic paradise from which we had been expelled, by drought and bankers, and could never return» $(1994, \text { p. 5) })^{12}$. La frontier tend donc à multiplier les objets de désir et à favoriser l'accès à ces objets et à contrôler la violence, sauf bien sûr face aux Autochtones. Le paradigme devient ainsi ternaire barbarie/civilisation/frontier et dégage un espace de coopération dans la rencontre de gens provenant de différentes régions de la planète et explorant les aléas comme les occasions fournies par la coïncidence spatiale.

\section{Métissage et réseautage}

La frontier ouvre potentiellement au métissage des espaces quand elle se transforme en lieu peuplé de créoles qui s'ouvrent à la modernité, car alors se combinent la spiritualité de l'infini à celle du Jardin d'Éden que l'on crée par l'application des savoirs de la modernité réalisant le plan de Dieu dans l'expansion de réseaux continentaux.

On mesure l'impact de ces réseaux chez le Canadien Anglais T. C. Keefer qui publie Philosophy of the Railroads en 1853. Il apporte des éléments sociologiques importants qui démontrent que les trains vont changer complètement le mode de vie des Canadiens en réduisant les distances qui se comptent maintenant en temps. C'est la durée qui devient importante et non l'espace en tant que tel comme le prouve le fait que les gens, dans les différentes régions du Canada, et partout progressivement dans les Amériques, vont s'habituer à vivre selon l'heure des fuseaux horaires et non selon l'heure locale. Voilà qui a pour corolaire, en 1885, le fait que le temps universel est établi à Greenwich en Angleterre, sous l'impulsion du Canadien Sandford Fleming. Le réseautage continental est en marche en attendant celui de la planète dont parle Pierre Lévy de nos jours (2011).

Ce progrès industriel, cette occupation active du territoire couplé à l'éducation et à l'exploitation du continent nécessitant l'arrivée massive d'immigrants est pensé par Randolph Bourne aux États-Unis en 1916, en termes de rencontres potentielles 
entre des groupes complémentaires comme on le voit dans le texte intitulé «Transnational America»:

What we have achieved has been rather a cosmopolitan federation of national colonies, of foreign cultures, from whom the sting of competition has been removed. America is already the world federation in miniature, the continent where for the first time in history has been achieved that miracle of hope, the peaceful living side by side, with character substantially preserved, of the most heterogeneous peoples under the sun. Nowhere else has such contiguity been anything but the breeder of misery ${ }^{13}$ (1916, p. 93).

La coupure entre barbarie et civilisation synonyme de campagne/ville ou de ville/ campagne suivant les optiques politiques est rejetée par Bourne et d'autres, mais elle va se maintenir face aux groupes minorisés comme les Autochtones ou les Noirs pendant encore longtemps, au moins jusqu'après la seconde guerre mondiale.

\section{La frontier et l'éden}

Même si elle peut prendre différentes valeurs, cette frontier est une catégorie importante pour les Amériques, car elle place le non-fini dans son fonctionnement culturel. Ce non-fini a toujours un lien avec une forme de pensée liée à la spiritualité. Elle est parfois directement métaphysique dans des conceptions propres à des groupes immigrants particuliers comme les Doukhobors. Gabrielle Roy le souligne dans la nouvelle intitulée «La vallée Houdou»: «Ils savent, ou à peu près, rapporta l'interprète, que la montagne et la rivière ne sont qu'illusion, mais ils disent: peu importe, puisque nous les voyons... Mais les Doukhobors ne voulaient rien entendre... assurés d'être les seuls à comprendre le mystère du monde...» (ROY, 1975, p. 147). Ce non-fini spirituel est philosophique de nos jours chez Yvon Rivard où la frontier est comparée à l'océan:

En effet, quand on marche dans la prairie, on a l'impression d'être à la surface, au milieu et au fond de quelque chose qui n'est ni la terre ni le ciel, mais un mélange des deux, une sorte d'océan à cause du ciel si haut qui pèse sur nous et de la lumière si pure qui gonfle le sol et allège notre pas (RIVARD, 2005, p. 303).

Cette frontier physique et métaphysique est explorée par le truchement d'un jeu comme le baseball ${ }^{14}$ qui est un espace vert à la fois clos et ouvert sur l'infini puisque la 
balle peut être frappée au-dessus de la clôture pour se perdre dans une frontier imaginaire qui évoque le paradis. On le note dans le roman Shoeless Joe Jackson comes to Iowa du Canadien W.P. Kinsella (1993). dans lequel Shoeless Joe Jackson, en parlant du champ des rêves, dit « This must be heaven » tandis que le narrateur Ray Kinsella réplique: «No. It’s Iowa» $»^{15}$ (Ibid., p. 43). Autrement dit, cette frontier du baseball qui a ouvert ses richesses aux immigrants est paradisiaque et à couleur d'un nouveau jardin d'Éden (TOWNER, 2007, p. 205-237). Elle est expression même de la Providence loin de l'Europe, ce que souligne fort pertinemment Georges Desmeules dans Le projet Syracuse: «Le baseball joue la mise à mort de l'Européen qui sommeille encore dans chaque Américain» (2008, p. 218). Cette providence fait rejoindre, dans le Nouveau Monde, tel que compris aux ÉtatsUnis, un discours d'une nouvelle naissance loin des conflits et de la pénurie, loin d'une logique territoriale où il faut non seulement travailler très dur, mais selon la logique du jeu à somme nulle, prendre aux autres une partie de la richesse pour survivre. Voilà qui nous fait rejoindre, quatre siècles plus tôt, le Jésuite Acosta ébloui de tant de beauté et de tant de richesses qui compare dans une envolée lyrique quasi hérétique, les Amériques au paradis:

Ceux qui pensent que le paradis terrestre est situé sous la zone équinoxiale, il semble que ce soit avec quelque raison. Non que je veuille décider par moi-même que le Jardin des Délices dont parle l'Écriture s'y trouve... mais parce que si l'on peut dire qu'il existe un paradis sur terre, c'est là où l'on jouit d'une température aussi suave et agréable (OUELLET, accessible sur: www.sociocritique. mcgill.ca/mythamerique.htm).

On pense aussi à Vespucci, cité par Delumeau: «[s]i le paradis terrestre subsiste en quelque endroit de la terre, j'estime... qu'il ne doit pas être très éloigné de ces régions» (DELUMEAU, 1992, p. 146). Mais si nous avons suivi le fil de l'espace ouvert colonisé, mis en valeur par les créoles des Amériques, fiers d'eux en cette frontier, il faut voir comment elle est recyclée au 20e siècle urbain dans un nouveau jardin, celui de la banlieue parsemée de bungalows fleuris comme va la décrire Jacques Ferron dans L'Amélanchier (1970). 


\section{De la frontier au bungalow}

Petit à petit, les grands espaces géographiques et immenses étant pleins, la frontier se transfère dans le monde industriel et urbain par le biais de la vision d'une richesse non finie à exploiter: le savoir et son application aux ressources naturelles. On le saisit déjà dans La Flore laurentienne du Frère Marie-Victorin (1935). Dans l'exploration des significations botaniques, il établit une compréhension nouvelle de l'environnement québécois et s'approprie le territoire et ses résonnances identitaires par la création d'un savoir. Par la suite, à partir du Frère Marie-Victorin, L'hiver de force de Réjean Ducharme (1973) cherche à relocaliser ce savoir dans les marges de la culture urbaine dominée par un accès au monde pris dans les stéréotypes médiatiques. Son intertexte propose de modifier le jardin en un espace urbain vivable où l'intimité contrôle la tristesse de l'existence urbaine ultra-réglée. Dans cette perspective, l'œuvre de Ducharme rappelle parfois L'Amélanchier de Ferron (1970) où le protagoniste crée un jardin édénique dans son arrière-cour (CULLER, 2004). Il ressemble à certains égards à l'intimité que propose le patio latino-américain. La cour arrière contraste avec la façade et avec le rythme trépidant et effréné de la rue et des parcs industriels adjacents et offre un substitut de lieu édénique.

Mais cette cour arrière est quand même restreinte et n'évite pas toujours les nostalgies plus profondes:

Every blade of grass, every trill from a red-winged blackbird, every sparkle of sun on the Frenchman River that trickled past our house at the hay farm seemed more precious... In the city for short visits now I studied people's houses, or the rows of condominiums or the new apartment buildings, assessing how close they were to each other, how big their yards were, what their occupants had for views, trying to imagine how I would live again in the city and what arrangement might be acceptable should something happen to Peter or the ranch... I tried to imagine life without this space, this welcome, close presence of grass and sky (BUTALA, 2000, p. 183).

Ces nostalgies qui obsèdent souvent les populations urbaines appellent, en plus de la cour arrière, l'art des calendriers et leur jeu sur la satisfaction d'avoir atteint un confort certain dans l'urbain tout en rêvant du coin de nature. Ici entrent en jeu le kitsch de Norman Rockwell et les paysages idylliques et stéréotypés des classes moyennes (MONSIVÁIS, 
1995, p. 67) recyclant les paysages romantiques du $19^{\mathrm{e}}$ siècle comme la petite maison au toit rouge perdue près de quelques arbres que l'on retrouve de l'Uruguay au Canada.

En tout cas, c'est dans les imaginaires de ces classes moyennes en mouvance que le bungalow et l'extension de la banlieue peuvent sembler apporter une solution agréable dans l'entre-deux nature/ville civilisée d'autant plus que la cour arrière du bungalow est suffisamment grande pour être transformée par les enfants en terrain de baseball, luimême relié au vrai terrain de baseball tout neuf du quartier issu de l'organisation spatiale des nouveaux rêves urbains construits à la campagne ${ }^{16}$ permettant d'échapper aux taudis ou aux espaces restreints du centre-ville. C'est d'ailleurs cette banlieue qui semble sans fin, logée entre ville et campagne, qui fait rêver les immigrants d'origine indienne avant de quitter diverses régions d'Afrique pour s'installer non pas à Toronto, mais à Mississauga. Cette banlieue est très connue sur la planète chez les aspirants à l'immigration comme le rappelle Arundhati Roy dans The God of Small Things (1997). Le rêve de l'expansion illimitée se niche dans un espace qui ne repose plus sur le dualisme ville/campagne ou civilisation/barbarie, mais qui les combine dans l'espoir de fonctionner selon la logique d'une nouvelle frontier celle d'un polyculturalisme lié à la capitalisation des savoirs et à des technologies ouvertes sur la nature de la cour arrière où le mâle chasseur est maître du barbecue. En effet, contrairement à Alejo Carpentier dans Los pasos perdidos (1979), il ne s'agit plus de se perdre dans la forêt profonde désormais traversée par la transamazonienne et habitée d'Autochtones qui sont délégués de diverses ONG (MATO, 1999, p. 129-147), pour remonter l'Orénoque et le temps, mais de gérer une infinité de connections dans de multiples réseaux mondiaux en les contrôlant le mieux possible, ce que montre Néstor García Canclini dans La globalización imaginada (1999).

Tout n'est pourtant pas édénique dans ce recyclage du jardin dans l'urbain. On le constate chez Jerzy Kosinski dans sa parodie de l'expulsion du Jardin d'Éden. Dans Being There (1971) Kosinski reformule ludiquement l'espace idyllique et clos du jardin. Il joue d'une façon humoristique avec l'intertexte d'œuvres littéraires consacrées au jardin. Being There, traduit en espagnol par Desde el Jardin, raconte l'histoire d'un jeune homme qui, complètement déconnecté de la société, demeure avec son tuteur dans une maison avec un magnifique jardin dont il est chargé de l'entretien. Sa seule autre activité est de regarder 
la télévision. Quand son tuteur meurt, le jeune homme, seul avec ses illusions créées par son existence réduite formée par le jardin et les discours médiatiques, est expulsé de la demeure. C'est alors qu'il découvre la ville pour la première fois. Mais il ne parle qu'en termes de jardinage. Les gens interprètent ses commentaires plutôt rudimentaires vis-àvis les saisons et le jardinage comme des métaphores correspondant à leurs stéréotypes de la modernité, de la vie urbaine et de l'importance du système économique. Les crises, dans le langage du héros vont s'en aller comme le printemps refleurit après un dur hiver. Ces métaphores éculées qui renvoient aussi à la structuration circulaire des romans de la terre que ce soit au Canada, aux États-Unis ou au Brésil17 , montrent l'imbrication des discours dans des changements de mode reliant la campagne à la ville, mais où le jardin édénique reste présent comme espoir de l'Amérique ${ }^{18}$. Ce personnage parvient en tout cas à se frayer un chemin dans cette nouvelle société et à devenir une vedette médiatique puis un des conseillers financiers du Président des États-Unis, car ses phrases sont lues comme des métaphores d'un mieux-être économique à venir et donc en fonction de l'expansion renouvelée de la frontier économique.

De toute évidence, Jerzy Kosinski propose une version du Jardin d'Éden non comme retour aux origines dans un espace clos et protégé, mais comme exploration de la nouvelle frontier qui, aux $20^{\mathrm{e}}$ et $21^{\mathrm{e}}$ siècles, n'est plus territoriale mais basée sur l'échange économique, la communication médiatique et l'accumulation des savoirs dans une urbanité en expansion globalisée. Dans cette frontier, des univers discursifs s'entrecroisent et génèrent de nouveaux espaces d'échanges dans une cacophonie compétitive mondialisée et démocratisée où les rapports de pouvoir se décontextualisent et se recontextualisent.

\section{Conclusion}

Cette frontier spatiale qui est aussi identitaire et dynamique et qui a attiré au $19^{\mathrm{e}}$ et au $20^{\mathrm{e}}$ siècles les immigrants dans les Amériques où l'élément révolutionnaire était de donner des titres de propriété aux pauvres est bien soulignée par Bharati Mukherjee dans Jasmine. On y observe le personnage $\mathrm{Du}$, un vietnamien adopté par une famille de l'Iowa après la conquête du Sud-Vietnam par les communistes et la collectivisation de la vie dans une dictature féroce: « owning is rebellion, it means not sharing, it means survival. He ate 
bugs and worms and rodents. He lived» (MUKHERJEE, 1989, p. 30) ${ }^{19}$. Du ne critique certes pas la propriété, la société de consommation ni la technologie, car c'est à cause de tout cela qui lui a manqué profondément que sa famille est morte et c'est grâce à tout cela qu'il va pouvoir recommencer. C'est aussi ce qu'analyse Doug Saunders dans son livre intitulé Arrival City où il se consacre à différents bidonvilles et banlieues inventés par des migrants et des immigrants qui créent des espaces urbains similaires partout dans le monde, notamment au Canada, aux États-Unis, au Brésil, au Venezuela. Il y montre la vitalité et le dynamisme des pauvres et des migrants qui sont pour lui non pas une plaie à contenir, mais l'avenir même des villes contemporaines: «These transitional spaces arrival cities - are the places where the next great economic and cultural boom will be born, or where the next great explosion of violence will occur. The difference depends on our ability to notice, and our willingness to engage $»^{20}$ (SAUNDERS, 2010, p. 3).

Cette dynamique issue d'une volonté de valoriser l'entreprise individuelle, montre que le paradigme barbarie/civilisation est couplé à de profondes structures de rejet qui proviennent des élites éduquées et des propriétaires terriens ou des bureaucrates urbains par rapport aux Métis, aux Autochtones et à tous les migrants et pauvres issus de l'intérieur ou de l'extérieur du pays. Ils finissent désormais par pénétrer les fonctionnements sociauxéconomiques et modifient radicalement le paysage des villes et même parfois des centres historiques contrôlés par les élites.

On voit que l'intégration des autres passe certes par la reconnaissance culturelle, mais aussi beaucoup par l'accès au travail et à l'économie, un des points fondamentaux de Arrival City de Doug Saunders et un point qui n'est pas assez mis en valeur par les théoriciens du multiculturalisme comme Charles Taylor ou Will Kymlicka qui soulignent toutefois que les droits culturels ne peuvent pas être reconnus efficacement sans que soient reconnus aussi les droits civils, sociaux et politiques. En ce qui concerne Saunders, on doit pourtant souligner que, dans ce contexte migratoire où les vies sont transformées, la lutte pour progresser économiquement est intense et les relations avec les communautés sont distendues, il reste à savoir comment les droits culturels peuvent être reconnus et reliés aux droits de citoyenneté alors que ce qui prime, c'est l'ambition de réussir et de 
migrer dans un environnement plus riche et confortable où la nouvelle frontier, la seule qui reste peut-être est celle qui ouvre sur la capitalisation de l'éducation et des savoirs.

Dans l'urbanité en expansion, celle qui, par le Web, Facebook, Twitter, internet, transforme même une maison isolée en pleine nature en foyer de rayonnement faisant partie du cerveau global dont parle Pierre Lévy (2007), tout est lieu de rencontres multiples. Ces rencontres ne sont plus contrôlées par les contraintes sociales propres aux lieux établis depuis longtemps, encore moins par un destin dépendant des dieux comme dans Antigone de Sophocle, mais par un hasard actif lié à des coïncidences nombreuses.

\section{Références}

ADIGA, Aravind. The White Tiger. New Delhi: HarperCollins Publishers India, 2008. ANDRADE, Oswald de. Manifeste anthropophage [1928]. Paris: Flammarion, 1982. AREA, Lelia. Una biblioteca para leer la Nación. Rosario, Argentina: Beatriz Viterbo Editora, 2006.

ALLENDE, Isabel. Hija de la fortuna. Buenos Aires: Editorial Sudamericana, 1999.

ATWOOD, Margaret. Survival. Toronto: Anansi, 1972.

BAEZ, Christian et MASON, Peter. "Zoológicos humanos" Fotografias de fueguinos y mapuches en el Jardin d'Acclimatation en Paris, siglo XIX. Pehuén Editores, 2006.

BERTON, Pierre. The National Dream: The Great Railway, 1871-1881. Toronto: McClelland and Steward, 1970.

BERTON, Pierre. The Last Spike: The Great Railway 1881-1885. Toronto: McClelland and Stewart, 1971.

BOUCHARD, Gérard et LACOMBE, Michel. Dialogue sur les pays neufs. Montréal: Boréal, 1999.

BOURNE, Randolph S. Trans-National America. The Atlantic Monthly, CXVIII, July 1916, p. 86-97.

BOURDÉ, Guy. Urbanisation et immigration en Amérique latine. Paris: Aubier, 1974.

BUTALA, Sharon. Wild Stone Heart. Toronto: HarperPerennial Canada, 2000.

BUTALA, Sharon. The Perfection of the Morning: an Apprenticeship in Nature. Toronto: HarperCollins Canada, 1994.

CANCLINI, García. La globalización imaginada. México /Buenos Aires /Barcelona: Paidos, 1999.

CARPENTIER, Alejo. Los pasos perdidos. Barcelona: Bruguera, 1979. 
CARRIER, Roch. Petit homme tornade. Montréal: Stanké, 1996.

CULLEN, Heather. L'herbier de force: l'intertextualité botanique chez Jacques Ferron et Réjean Ducharme. Thèse de maîtrise. Département des lettres françaises. Université d'Ottawa, 2004.

DELUMEAU, Jean. Une histoire du paradis. Paris: Fayard, 1992.

DESMEULES, Georges. Le projet Syracuse. Vie et mort de Wolf Habermann (1985 ? - 1979 ?), mathématicien, philologue, amateur de baseball et soi-disant conspirateur [2008]. Québec : L'instant même, 2011.

DUCHARME, Réjean. L'hiver de force. Paris: Gallimard, 1973.

FERRON, Jacques. L'Amélanchier. Montréal: Éditions du Jour, 1970.

FRÈRE MARIE-VICTORIN. La Flore laurentienne. Montréal: La Salle, 1935.

FRESONKE, Kris. West of Emerson: The Design of Manifest Destiny. Berkeley: University of California Press, 1999.

GERMAIN, Georges-Hébert (sous la direction scientifique de David Morrison). Inuit: Les peuples du froid. Ottawa: Musée canadien des civilisations, 1995.

IMBERT, Patrick. «Destino Manifesto» In: Zilá BERND (Org.). Dicionário de Figuras e Mitos Literários das Américas. Porto Alegre: Universidade Federal do Rio Grande do Sul \& Tomo Editorial, 2007.

IMBERT, Patrick. Les Amériques transculturelles: les stéréotypes du jeu à somme nulle. Québec: Presses de l'Université Laval, 2013.

IMBERT, Patrick. «Cultural Changes and Economic Liberalism in Canada and in the Americas». In: Converging Disensus? Cultural Transformations and Corporate Cultures: Canada and the Americas. Ottawa, University of Ottawa Research Chair: «Canada: Social and Cultural Challenges in a Knowledge-Based Society» Publisher, 2006, p. 15-77.

IMBERT, Patrick. L'Interculturalism. Interfaces Brasil/Canadá, v. 14, n. 1, p. 27-41, 2014.

KINSELLA, W. P. Shoeless Joe Jackson comes to Iowa. Dallas: Texas, Southern Methodist University Press, 1993.

KOSINSKI, Jerzy. Being There. New York: Harcourt Brace Jovanovich, 1971.

LACASSE, Jean-Paul, Les Innus et le territoire: Innu tipenitamun. Sillery (Québec): Septentrion, 2004.

LANGUIRAND, Jacques. Klondyke suivi de Le Québec et l'Américanité. Montréal: Cercle du livre de France, 1971.

LÉVY, Pierre. La sphère sémantique I: computation, cognition, économie de l'information. Paris: Lavoisier, 2011.

LÉVY, Pierre. «Société du savoir et développement humain». In: Patrick Imbert (Dir.), Le Canada et la société des savoirs: Le Canada et les Amériques. Ottawa: Chaire de 
recherche de l'Université d'Ottawa: Enjeux sociaux et culturels dans une société des savoirs, 2007.

MATO, Daniel. Sobre la fetichización de la globalización y las dificultades que plantea para el estudio de las transformaciones sociales contemporâneas. Revista venezolana de analísis de coyuntura, v. V, n.1, janvier-juin 1999, p. 129-147.

MICHEAUX, Oscar. The Conquest: The Story of a Negro Pioneer. Lincoln, Nebraska: The Woodruff Press, 1913.

MONSIVÁIS, Carlos. Los rituales del caos. México: Era, 1995.

MORRISON, David and GERMAIN, Georges-Hébert. Inuit: Glimpses of an Arctic Past. Ottawa: Canadian Museum of Civilization, 1995.

MORTON, W. L. The North in Canadian Historiography. In: A.B. McKillop (Ed.), Contexts of Canada's Past: Selected Essays of W. L. Morton. Toronto/Ottawa: Macmillan/ Carleton University Press, 1980, p. 229-239.

MUKHERJEE, Bharati. Jasmine. New York: Grove and Weidenfeld, 1989.

NAREAU, Michel. Double jeu: baseball et littératures américaines. Montréal : Le Quartanier, 2012.

OUELLET, Réal. Locus amoenus, âge d'or et paradis terrestre dans les textes français du XVIIle siècle sur les Antilles. Accessible sur: www.sociocritique.mcgill.ca/mythamerique. htm, consulté en 2005.

PALEY, William. Natural Theology or Evidences of the Existence and Attributes of the Deity. London: J. Faulder, 1809.

KEEFER, T. C. Philosophy of the Railroads. Montréal: Lovell, 1853.

REIMERS, Camila. Tres lotos en un mar de fuego. Ottawa: Art and Literature Mapalé and Publishing Inc., 2006.

RIVARD, Yvon. Le siècle de Jeanne. Montréal: Boréal, 2005.

RIZZO, Adriana. Exclusion sociale et exclusion de l'immigrant en Argentine aux temps de la mondialisation. Leurs traces dans les discours médiatiques. In: Daniel Castillo Durante et Patrick Imbert (Dir.). L'interculturel au cœur de Amériques. Collection des Amériques/ The Americas Series/Colección de las Américas, Université d'Ottawa/Université du Manitoba/Legas, 2003, p. 143-166.

ROY, Arundhati. The God of Small Things. Toronto: Random House of Canada, 1997.

ROY, Gabrielle. La vallée Houdou. In: Un jardin au bout du monde. Montréal:

Beauchemin, 1975.

SARMIENTO, Domingo Faustino. Facundo. [1845]. Paris: Stock, 1934.

SAUNDERS, Doug. Arrival City. Toronto: Knopf, 2010.

SOTO, Hernando de. The Other Path: The Invisible Revolution in the Third World. New 
York: Harper and Row, 1989.

TOWNER, Marc-André. Le jardin de baseball des Amériques. In: Patrick Imbert (Dir.).

Les jardins des Amériques : éden, home et maison. Ottawa: chaire de recherche: Canada: enjeux sociaux et culturels dans une société du savoir, 2007, p. 205-237.

TURNER, Frederick Jackson. The Significance of the Frontier in American History, 1893. In: Rereading Frederick Jackson Turner. Ed. John Mack Faragher. New York: Holt, 1994.

\section{Notes}

1 Professeur éminent, membre de la Faculté des Études Supérieures et Postdoctorales, Département de Français, Université d'Ottawa, Ontario, Canadá. pimbert@uottawa.ca.

2 Évidemment, là aussi l'espace est inventé par des discours comme le rappelle Sharon Butala dans Wild Stone Heart: «The more I think about the Sasquatch as non-Amerindians understand him, the more I think of him as an inevitable construct for a people arriving on a new continent about which they knew nothing, refusing to learn from its inhabitants, continuing to declare the continent as 'empty' and 'untouched', despite the evidence of its far-flung population whose ancient ability to survive on the land's resources should have been obvious to the new arrivals» (2000, p. 121). Nous traduisons: Plus je pense au Sasquatch, à la manière dont les non-amérindiens le comprenne, plus je pense à lui comme une construction inévitable de la part d'un peuple arrivant dans un nouveau continent au sujet duquel ils ne savent rien, refusant d'apprendre de ses habitants, continuant à déclarer le continent 'vide' et 'inviolé' et cela en dépit de l'évidence de sa population étendue dont la capacité ancienne de survivre sur les ressources du territoire aurait dû être évidente aux nouveaux arrivants.

3 Nous traduisons: Au moment où la frontier agricole s'est déplacée vers l'Ouest, elle a laissé un flanc ouvert au nord. Cela est devenu une frontière permanente, une ligne de démarcation stable entre l'espace sauvage et l'espace agricole, entre le Nord et le Sud.

4 Nous traduisons: Finalement, le Nord... comme source de trésors... le développement d'une frontier permanente.

5 Nous traduisons: Le héros collectif peut être l'expression d'une mentalité de garnison fermée et incestueuse, ou bien d'une communauté vivante; l'action collective a été nécessaire pour survivre mais elle peut aussi étouffer le développement individuel.

${ }^{6}$ Nous traduisons: Le Nord rend nécessaire une dépendance absolue de ses compagnons, d'habiletés coopératives et de capital communautaire.

7 Nous traduisons: Conversation idéale dans les ascenseurs, les fêtes diplomatiques et le commencement des réunions commerciales. Plus il fait froid, mieux c'est, nous nous sentons unis par une nature inhospitalière, nous sommes des héros partageant un destin congelé.

8 Nous traduisons: L'Ouest a ouvert un refuge par rapport aux règles des classes établies, à la subordination des jeunes soumis aux vieux et à l'influence des institutions établies et révérées.

9 De plus, en Argentine, en 1876, une loi inspirée par le Homestead Act des États-Unis permet la distribution de lots de 25 à 50 hectares aux immigrants. Néanmoins, rapidement d'immenses étendues du « désert » sont octroyées aux individus de la classe dirigeante, si bien qu'en 1890 rien ne resta plus à donner. Voir : BOURDÉ, 1974, p. 151.

10 Même chose pour les italiens colonisant 50 ans après les Allemands, les régions autour de Porto Alegre dans le sud du Brésil, où ils transforment la région en foyer de production vinicole.

11 Nous traduisons : La Providence a inondé de bienfaits sans nombre ce territoire favorisé de ses bontés et vous a fait les gardiens de la liberté pour en faire bénéficier la race humaine.

12 Nous traduisons : C'est ainsi que mes sœurs et moi avons grandi dans l'idée que la ferme était un paradis mythique dont nous avons été chassées, par la sécheresse et les banquiers, et où nous ne pourrions jamais retourner.

13 Nous traduisons: Ce que nous avons atteint est plutôt une fédération cosmopolite de colonies nationales, de cultures étrangères au sujet desquelles la piqûre de la compétition a été retirée. L'Amérique est déjà la fédération mondiale en miniature, le continent où, pour la première fois dans l'histoire, a été atteint ce miracle de l'espoir, le fait de vivre côte à côte et en paix, avec des caractéristiques substantiellement 
préservées, des peuples les plus hétérogènes qui vivent sous le soleil. Nulle part ailleurs une telle contiguïté n'a été autre chose qu'un porteur de misère.

${ }^{14}$ Pour voir la présence importante de ce jeu dans la littérature: NAREAU, Michel, Double jeu: baseball et littératures américaines, Montréal, Le Quartanier, 2012.

${ }^{15}$ Nous traduisons: Ça doit être le paradis... Non c'est l'Iowa.

${ }^{16}$ Voir: TOWNER, 2007, p. 205-237.

${ }^{17}$ Ils reposent aussi sur une causalité païenne autochtone en parallèle avec la linéarité liée à l'Église catholique allant du Jardin d'Éden au paradis.

${ }^{18}$ Évidemment cet espoir tourne parfois au très sombre comme chez Philip Roth dans American Pastoral (1997).

19 Nous traduisons: Posséder c'est se rebeller, cela signifie ne pas partager, cela signifie survivre. Il a mangé des insectes, de vers et des rongeurs. Il a vécu.

${ }^{20}$ Nous traduisons: Ces espaces transitionnels - les villes d'arrivées - sont les lieux où va naître le prochain grand développement économique et culturel, ou bien où la prochaine grande explosion de violence aura lieu. La différence dépend de notre capacité à le remarquer et de notre volonté d'y participer. C'est aussi ce que suggère Aravind Adiga dans The White Tiger (2008): «It may turn out to be a decent city, where humans can live like humans and animals can live like animals. A new Bangalore for a new India. And then I can say that, in my own way, I helped to make New Bangalore». Nous traduisons : Elle pourrait devenir une ville décente où les humains peuvent vivre comme des humains et les animaux peuvent vivre comme des animaux. Une nouvelle Bangalore pour une Inde nouvelle. Et alors je peux dire que, à ma façon, j'ai aidé à faire la Nouvelle Bangalore.

${ }^{21}$ On voit des situations similaires produites par la mondialisation et l'influence de la valorisation des idées d'entreprenariat et d'affirmation individuelle dans le roman de Aravind Adiga, The White Tiger (2008). On le voit aussi dans de nombreux articles publiés dans le magazine Wired montrant l'évolution de la culture en Inde sous l'effet de l'accès à l'éducation et de l'ouverture au monde (IMBERT, 2006, p. 15-77). 